\title{
Contribution of the ulnar digits to grip strength
}

\author{
Jennifer Methot BHK MScOT ${ }^{1,2}$, Shrikant J Chinchalkar MThO BScOT OTR CHT OTReg (Ont) ${ }^{2}$, Robert S Richards MD FRCSC 1,2
}

\begin{abstract}
J Methot, SJ Chinchalkar, RS Richards. Contribution of the ulnar digits to grip strength. Can J Plast Surg 2010;18(1):e10-e14.
\end{abstract}

PURPOSE: To determine the contribution of ulnar digits to overall grip strength.

SUBJECTS: Fifty individuals ( 25 men and 25 women; 100 hands) with a mean age of 35.6 years (range 19 to 62 years) were tested. Exclusion criteria included previous history of hand injuries, entrapment neuropathies and systemic diseases.

METHODS: Ethics approval was granted before testing. A calibrated Jamar dynamometer (Lafayette Instrument Company, USA) was used to test subjects in three configurations: entire hand - index, middle, ring and little fingers; index, middle and ring fingers; and index and middle fingers. Little and ring fingers were excluded using generic hand-based finger splints. The order of testing was kept constant, and subjects were tested three times on each hand for each configuration. The average of the three trials at each configuration was recorded. Subjects received 1 min of rest between each testing configuration. The data were analyzed using a $3 \times 2$ repeated measures ANOVA with hand dominance and configuration as the within-subject factors, followed by two independent sample $t$ tests to compare flexor digitorum superficialis (FDS) independence and FDS nonindependence on right and left hand grip strength measurements in the index, middle, ring and little condition.

RESULTS: Univariate results demonstrated that grip strength was significantly predicted by the interaction between hand dominance and configuration, while the parsing of the interaction term demonstrated greater grip strength across all levels of configuration for the dominant and nondominant hand. There were no significant differences between FDS independence and FDS nonindependence for either hand on grip strength.

DISCUSSION: The results indicate a significant decrease in grip strength as ulnar fingers were excluded. Furthermore, exclusion of the little finger has differing effects on the grip strength of the dominant and nondominant hands - the dominant hand had a greater loss of strength with the little finger excluded than the nondominant hand.

CONCLUSIONS: The ulnar two digits play a significant role in overall grip strength of the entire hand. In the present study, exclusion of the ulnar two digits resulted in a $34 \%$ to $67 \%$ decrease in grip strength, with a mean decrease of $55 \%$. Exclusion of the little finger from a functional grip pattern decreased the overall grip strength by $33 \%$. Exclusion of the ring finger from a functional grip pattern decreased the overall grip strength by $21 \%$. It is clear that limitation of one or both of the ulnar digits adversely affects the strength of the hand. In addition, there was no significant difference between grip strength of FDSindependent and FDS-nonindependent subjects for either hand.

Key Words: Grip strength; Testing; Ulnar digits

\section{Contribution des doigts ulnaires à la force préhensile}

BUT : Déterminer la contribution des doigts ulnaires à la force globale de préhension.

SUJETS : Cinquante personnes ( 25 hommes, 25 femmes, 100 mains) âgées en moyenne de 35,6 ans (de 19 à 62 ans) ont subi les tests. Les critères d'exclusion incluaient les antécédents de blessures aux mains, les syndromes canalaires et les maladies systémiques.

MÉTHODES : Les auteurs ont reçu l'approbation du comité d'éthique avant de procéder aux tests. Ces derniers ont été effectués avec un dynamomètre Jamar étalonné (Lafayette Instrument Company, É.-U.) selon trois configurations : main entière - index, majeur, annulaire et auriculaire, index, majeur et auriculaires, et index et majeur. Les auriculaires et les annulaires ont été immobilisés au moyen d'attelles digitales génériques reliées à la main. Les tests ont tous été faits dans le même ordre et les sujets ont subi trois tests pour chaque main et chaque configuration. Les auteurs ont calculé la moyenne des trois tests de chaque configuration. Les sujets avaient une pause d'une minute entre chaque configuration testée. Les données ont été soumises à une ANOVA 3 x 2 à mesures répétées avec comme facteurs intra-individuels la latéralité (main dominante) et la configuration, suivie de deux tests t à échantillons indépendants pour comparer l'indépendance et la non-indépendance des fléchisseurs superficiels des doigts (FSD) dans la force de préhension de la main droite et de la main gauche pour l'index, le majeur, l'annulaire et l'auriculaire.

RÉSULTATS : Les résultats univariés ont démontré que l'interaction entre la latéralité et la configuration était un prédicteur significatif de la force de préhension, tandis que l'analyse fine l'interaction a révélé que la force de préhension était plus marquée dans tous les niveaux de configuration pour la main dominante et nondominante. On n'a noté aucune différence significative entre l'indépendance et la non-indépendance des FSD d'une main à l'autre pour ce qui est de la force préhensile.

DISCUSSION : Les résultats indiquent une baisse significative de la force de préhension lorsque les doigts ulnaires sont exclus. En outre, l'exclusion de l'auriculaire exerce des effets différents sur la force préhensile de la main dominante et de la main non-dominante, la main dominante perdant davantage de force que la main non-dominante lorsque l'auriculaire est exclu.

CONCLUSION : Les deux doigts ulnaires jouent un rôle significatif dans la force globale de préhension de la main entière. Dans la présente étude, l'exclusion des deux doigts ulnaires a donné lieu à une diminution de $34 \%$ à $67 \%$ de la force préhensile (moyenne $55 \%$ ). L'exclusion l'auriculaire pour le mode de préhension fonctionnel a réduit la force globale de préhension de $33 \%$. L'exclusion de l'annulaire pour le mode de préhension fonctionnel a réduit la force globale de préhension de $21 \%$. Il est clair que toute restriction impliquant un des doigts ulnaires ou les deux affaiblit la main. De plus, on n'a noté aucune différence significative entre la force de préhension des sujets selon l'indépendance ou la non-indépendance des FSD au niveau des deux mains.

On the other hand, our clinical experience has shown that patients with injuries involving the ulnar side of the hand exhibit great losses in overall grip strength. While research has explored individual digit strength, each digit does not act alone. All digits and muscles must work together in a coordinated fashion to produce overall grip strength. Further to this, muscle contraction in one finger has been shown to generate tension in other fingers (4). Activation of muscle units in the little finger also produced relatively large forces under the ring finger, suggesting that the little finger contributes significantly to the force production under the ring finger (4). Bowman et al (5) measured the impact of the flexor digitorum superficialis (FDS) of the little strength differences between the middle and little fingers.

${ }^{1}$ University of Western Ontario; ${ }^{2}$ Hand $\mathcal{E}$ Upper Limb Centre, St Joseph's Health Care, London, Ontario

Correspondence: Dr Robert S Richards, Division of Plastic Surgery, University of Western Ontario, Hand $\mathcal{E}$ Upper Limb Centre,

St Joseph's Health Care, London, Ontario N6A 4A6. Telephone 519-646-6046, fax 519-646-6049, e-mail rrichard@uwo.ca 

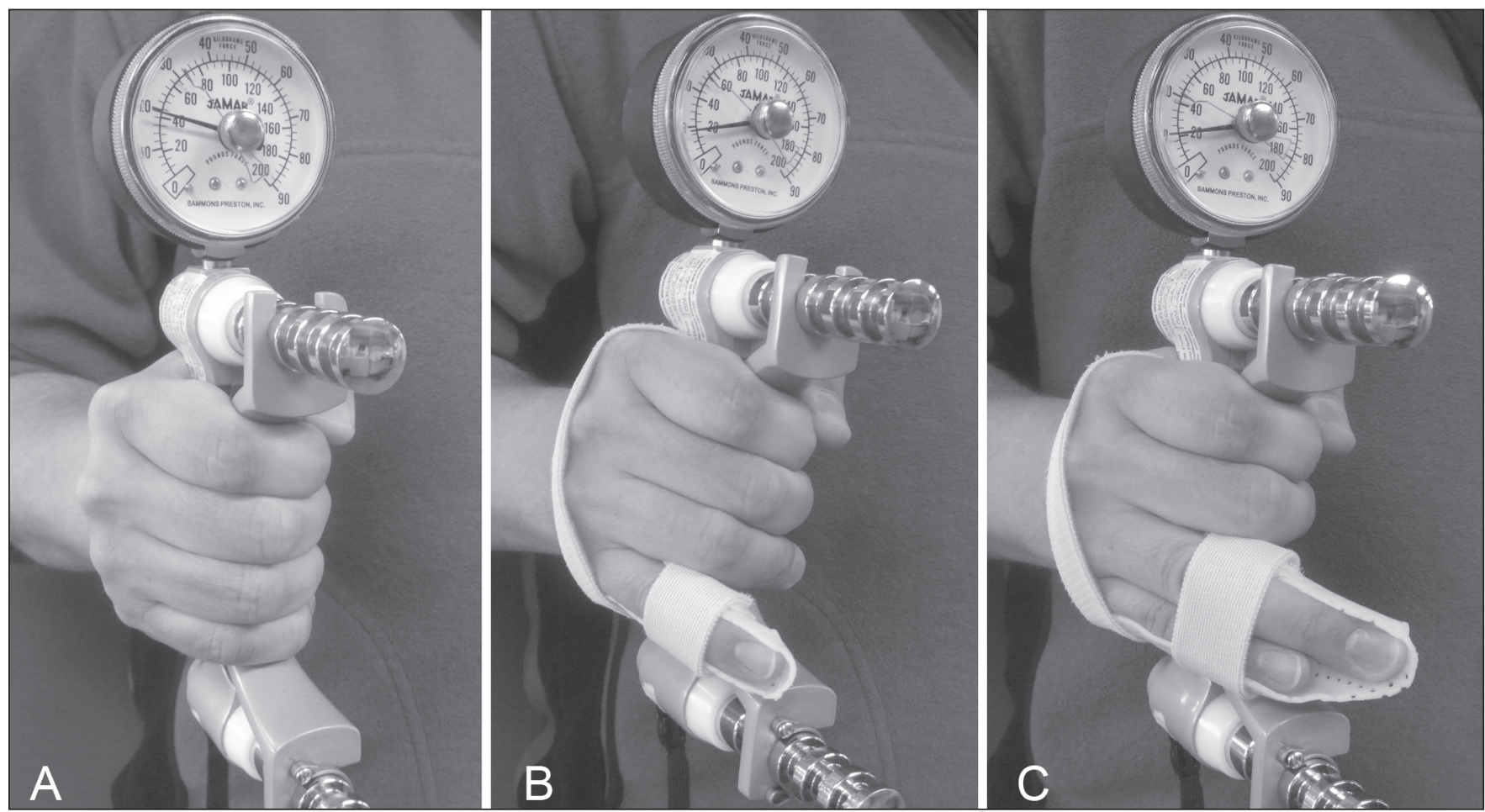

Figure 1) Configurations of grip strength testing

finger on grip strength and found that subjects with nonindependent little finger FDS had significantly weaker grip strength than FDS-independent subjects.

The flexor digitorum profundus muscle has a common belly and separates distally in the forearm to supply a single tendon to each finger $(4,6)$. Therefore, independent control of each finger may be limited by the degree to which the muscles were "subdivided into separate anatomical compartments controlling each finger" (4). Interconnection among the muscle fibres acting on all of the digits maximizes the force output during grip strength testing. In the FDS muscle, independent muscle bellies also act on each digit. Any limitation in joint motion or decreased excursion of the tendons going to any one finger can cause a decrease in maximal force output from that individual digit. During flexion, such as that occurring during gripping, the lumbricals may dominate the closure sequence, coordinating the closure of the hand around an object (7). Overall grip strength is a result of the interaction of many muscles originating from the forearm or hand and inserting into the hand and fingers.

The present paper examines overall grip strength when the ulnar digits are restricted. The purpose of the study is to determine the contribution of the little finger and the little and ring finger together in overall grip strength. It is hypothesized that the ulnar digits make a significant contribution to functional grip strength. A secondary hypothesis is that subjects with nonindependent little finger FDS will display significantly weaker grip strength than subjects with independent little finger FDS.

\section{METHODS}

Subjects

Participants were selected using a convenience sample of hospital staff and university students. The sample included 50 hands from 25 men (21 right hand dominant; four left hand dominant) with an age range between 20 and 62 years (mean \pm SD
$37.84 \pm 12.85$ years), and 25 women ( 23 right hand dominant; two left hand dominant) with an age range between 19 and 58 years (mean \pm SD $32.12 \pm 10.95$ years). Exclusion criteria included history of hand injuries, entrapment neuropathies and systemic diseases. Ethics approval for the project was granted before testing these individuals.

\section{Study design and procedures}

A calibrated Jamar dynamometer (Lafayette Instrument Company, USA) with an adjustable handle was used to test all subjects. This type of dynamometer is often used in rehabilitation settings and is supported with published test protocols. The Jamar dynamometer has five settings representing grip spans of $1.0,1.5,2.0,2.5$ and 3.0 inches. To obtain maximal grip strength, the second setting is the recommended one for both clinical and research purposes (8). All testing in the study was completed on the second setting (1.5 inch grip span). There are no published data to indicate any effect of handle shape on position of nonexcluded fingers.

Subjects were tested in three configurations: index, middle, ring and little fingers; index, middle and ring fingers; and index and middle fingers. Subjects were tested in a seated position with the shoulder adducted and neutrally rotated, the elbow flexed to 90 degrees, and the forearm and wrist in a neutral position in accordance with the standardized testing position suggested by the American Society of Hand Therapists $(9,10)$. The excluded fingers were held in place by generic splints. The splints held the metacarpal phalangeal joint in 45 degrees flexion, the proximal interphalangeal joint in 30 degrees flexion and the distal interphalangeal joint in a neutral position. Figure 1 demonstrates the configurations.

The order of testing was kept constant, and the subjects were tested three times on each hand for each configuration. Subjects rested for $1 \mathrm{~min}$ between trials. Subjects were also 
TABLE 1

\section{Descriptive statistics}

\begin{tabular}{|c|c|c|c|c|c|}
\hline Configurations & $\mathbf{n}$ & Median & Minimum & Maximum & Mean \pm SD \\
\hline Dominant index, middle, ring, little & 50 & 44.33 & 21.67 & 66.00 & $43.21 \pm 12.30$ \\
\hline Dominant index, middle, ring & 50 & 34.67 & 11.33 & 46.00 & $28.14 \pm 8.95$ \\
\hline Dominant index, middle & 50 & 26.67 & 10.00 & 36.67 & $20.13 \pm 6.38$ \\
\hline Nondominant index, middle, ring, little & 50 & 45.00 & 21.33 & 66.33 & $40.47 \pm 11.94$ \\
\hline Nondominant index, middle, ring & 50 & 38.00 & 12.33 & 50.33 & $27.59 \pm 9.36$ \\
\hline Nondominant index, middle & 50 & 23.33 & 9.33 & 32.67 & $18.48 \pm 5.94$ \\
\hline
\end{tabular}

tested for an independent or nonindependent little finger FDS. The testing procedure took approximately $20 \mathrm{~min}$ to complete. The average of the three trials at each configuration was recorded and analyzed.

\section{Data analysis}

The data were analyzed using a $3 \times 2$ repeated measures ANOVA with hand dominance (dominant and nondominant) and configuration (index, middle, ring and little fingers; index, middle and ring fingers; and index and middle fingers) as the within-subject factors. Significant interaction terms were further parsed by examining simple main effects. All univariate analyses were evaluated using the Greenhouse-Geisser epsilon correction for lack of sphericity. Posthoc evaluations of significant main effects were conducted using Bonferroni-adjusted pairwise comparisons. Effect size measures were presented as partial eta-squared, which can be interpreted as the percentage of variance accounted for by an effect after controlling for other factors in the model. In addition, two independent sample $t$ tests were run to compare FDS independence and FDS nonindependence on right and left hand grip strength measurements in the index, middle, ring and little condition.

\section{RESULTS}

Table 1 presents the range and mean \pm SD for the three conditions by dominant and nondominant hand. Interestingly, a large variability existed among the SDs across the conditions, such that, as the total digits contributing to grip strength decreased, so did the total variability across participants. This effect was consistent across dominant and nondominant hands.

Univariate results demonstrated that grip strength was significantly predicted by the interaction between hand dominance and configuration $(F[1.95,95.55]=8.46 ; \mathrm{P}<0.001)$. The mean plot for the interaction term is displayed in Figure 2. Similarly, significant main effects were demonstrated for both hand dominance $(F[1,49]=13.56 ; \mathrm{P}<0.001)$ and grip configuration ( $F[1.30,63.82]=415.33 ; \mathrm{P}<0.001)$. The significant interaction term was parsed by evaluating the effect size for grip configuration at each level of hand dominance. These results, presented in Table 2, demonstrate that the effect of configuration on grip strength was greatest when using the dominant hand.

Posthoc evaluation of the simple main effect of configuration yielded a statistically significant difference between each level of grip configuration, with grip strength decreasing as the number of digits contributing decreased. This pattern of effect was consistent across both the dominant and the nondominant hand. Posthoc evaluation of the simple main effect of hand dominance yielded a statistically significant difference in grip strength between the dominant and nondominant hand, with the dominant hand demonstrating greater overall grip strength.

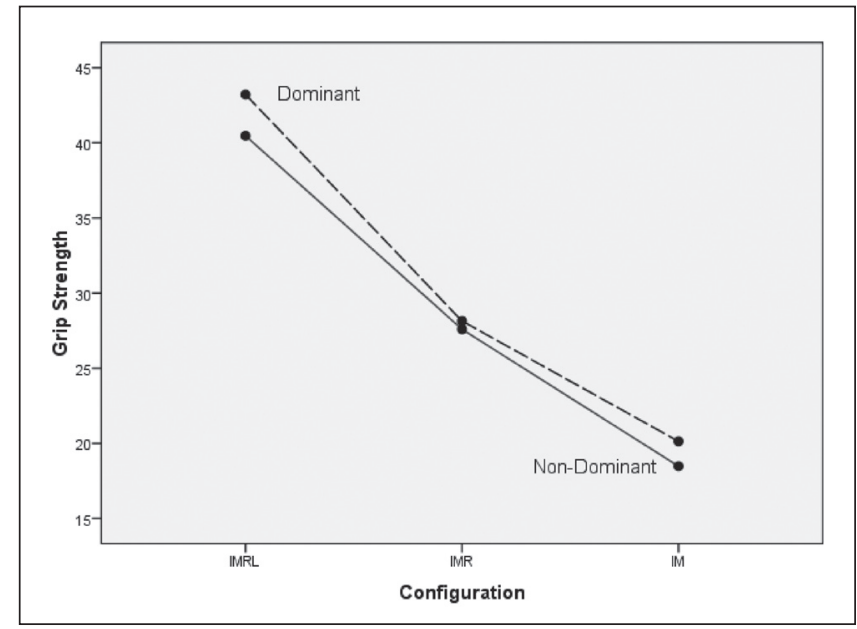

Figure 2) Effect of configuration on grip strength. IMRL Index, middle, ring, little

The abovementioned posthoc comparisons are presented in Table 3.

\section{Little finger FDS}

Given that hand dominance has a significant effect on overall grip strength, only participants who were right hand dominant were selected for this analysis to remove hand dominance as a confounding factor. The number of left hand dominant participants was insufficient to allow for meaningful interpretation of the data. Forty-four participants were right hand dominant, 29 were FDS independent and 15 were FDS nonindependent for both hand conditions. In the comparison of FDS independence and FDS nonindependence for the right hand, Levene's test demonstrated no significant violation of homogeneity between groups $(\mathrm{P}=0.64)$; therefore, equal variances were assumed for further interpretation. There were no significant differences between groups for the right hand on the grip strength variable $(t[42]=-0.19, \mathrm{P}=0.85)$. Next, FDS independence and FDS nonindependence were compared for the left hand, again assuming equality of variances as per Levene's test $(\mathrm{P}=0.82)$. There were no significant differences between groups for the left hand on the grip strength variable $(t[42]=0.11 ; \mathrm{P}=0.92)$.

\section{DISCUSSION}

The results of the present study indicate a significant decrease in grip strength as fingers were excluded. Grip strength decreased by an average of $33 \%$ with the little finger restricted, and an average of $54 \%$ with both the little and ring finger restricted. However, exclusion of the little finger had differing effects on the grip strength of the dominant and nondominant hands, because the dominant hand had a greater loss of strength with 
TABLE 2

Univariate effects of configuration under each level of dominance

\begin{tabular}{|c|c|c|c|c|c|c|}
\hline & \multicolumn{3}{|c|}{ Dominant } & \multicolumn{3}{|c|}{ Nondominant } \\
\hline & $\boldsymbol{F}$ & d.f. & $\mathrm{Pt} \eta^{2}$ & $\boldsymbol{F}$ & d.f. & $P t \eta^{2}$ \\
\hline Grip strength & 403.79 & $1.33,65.22$ & 0.892 & 344.33 & $1.42,69.72$ & 0.875 \\
\hline
\end{tabular}

d.f. Degrees of freedom; Pt $\eta^{2}$ Partial eta-squared

TABLE 3

Bonferroni-adjusted pairwise comparisons

\begin{tabular}{lcccc}
\hline & & & \multicolumn{2}{c}{$\mathbf{9 5 \%} \mathbf{~ C l}$ for difference } \\
\cline { 3 - 5 } Comparison & Mean difference & $\mathbf{P}$ & Lower bound & Upper bound \\
\hline IMRL - IMR & 13.97 & 0.001 & 12.164 & 15.783 \\
IMR - IM & 8.56 & 0.001 & 7.246 & 9.874 \\
IMRL - IM & 22.53 & 0.001 & 19.987 & 25.080 \\
D - N & 1.65 & 0.001 & 0.749 & 0.2549 \\
\hline
\end{tabular}

D Dominant; IMRL Index, middle, ring, little; N Nondominant

the little finger excluded than the nondominant hand. Deficiency of the little finger FDS did not have a significant effect on overall grip strength.

In the past, individual digit strength was measured using digital hand-held dynamometers or other force transducer devices $(11-13)$. It is difficult to use these results clinically because they are not directly comparable with our study in which the Jamar dynamometer was used. Based on individual digital measurements, MacDermid et al (2) suggested the ulnar digits (little and ring fingers) contribute a lesser proportion of overall grip strength. Digital contributions to overall grip strength have been approximated at 25\%,35\%, 26\% and 15\% for the index, long, ring and little fingers, respectively $(1,2)$. However, forces produced by the individual digit do not act only on that digit and do not act in isolation. In the current study, grip strength significantly decreased when the ulnar digits were excluded. Exclusion of the little finger from a functional grip pattern decreased the overall grip strength by $33 \%$. Exclusion of the ring finger from a functional grip pattern decreased the overall grip strength by $21 \%$. Exclusion or neutralization of both ring and little fingers gave a loss of $54 \%$ grip strength. However, this does not suggest that the contribution of the index and middle fingers in a normal hand would be equal to $46 \%$ grip strength. When compared with past research measuring individual digital strength, it is clear that the little finger is an important contributor to overall grip strength beyond individual digital strength. This would appear to support past research indicating that activation of little finger motor units also cause tension in other fingers (4).

Furthermore, hand dominance had a significant interaction effect with grip configuration, with the dominant hand experiencing a greater loss of strength when the little finger was excluded than the nondominant hand. Functionally, this is important because it suggests that individuals experiencing injury of the dominant hand will suffer greater strength loss resulting in large functional deficits in the dominant hand.

Regardless of individual digital strength in either hand, loss of the use of one or both of the ulnar digits will have significant functional impact and will require treatment to regain strength and function. Our results suggest that one should expect individuals with ulnar digit injuries to exhibit a significant loss of overall grip strength and a significant negative impact on functional activities.
One drawback of the study is that the testing procedure was not randomized: each subject was tested in the same order beginning with whole hand grip strength, then little finger excluded, and finally both little and ring fingers excluded. Fatigue could have confounded the results, contributing to some of the decrease in strength in the second and third configurations. A follow-up study should be conducted with a randomized testing procedure to control for the effects of fatigue. Another limitation is that grip strength was only measured in a static grip strength position. Static grip strength measurement may not represent functional strength of the hand as well as dynamic grip strength (DGS). In DGS, stabilization is decreased resulting in submaximal grip strength. It is suggested that DGS may more accurately represent functional activities $(9,14)$. The contributions of the ulnar digits may differ in DGS, which would impact functional activities because the wrist and forearm often move through a range of motion. Other factors limiting the results of our study include variable limitation in joint motion; reduced excursion and its effect on strength were not measured. Simulation of this kind and collection of data are near impossible.

\section{CONCLUSION}

The ulnar two digits play a significant role in overall grip strength of the entire hand. In our study, exclusion of the ulnar two digits resulted in up to a $54 \%$ decrease in grip strength. Exclusion of the little finger from a functional grip pattern decreased the overall grip strength by $33 \%$. Exclusion of the ring finger from a functional grip pattern decreased the overall grip strength by $21 \%$. It is clear that limitation of one or both of the ulnar digits will adversely affect hand function. More research is needed to truly understand the contributions of the ulnar digits to grip strength. Future studies should explore the effect of randomizing the testing protocol and the effect of restriction of the ulnar digits in a dynamic grip test situation.

\section{REFERENCES}

1. Talsania JS, Kozin SH. Normal digital contribution to grip strength assessed by a computerized digital dynamometer. J Hand Surg (Br) 1998;23:162-6.

2. MacDermid JC, Lee A, Richards RS, Roth JH. Individual finger strength: Are the ulnar digits "powerful"? J Hand Ther 2004:17:364-7.

3. Ejeskar A. Ortengren R. Isolated finger flexion force a methodological study. Hand 1981;13:223-30.

4. Kilbreath SL, Gorman RB, Raymond J, Gandevia SC. Distribution of the forces produced by motor unit activity in the human flexor digitorum profundus. J Phys 2002;543:289-96.

5. Bowman P, Johnson L, Chiapetta A, Mitchell A, Belusko E. The clinical impact of the presence or absence of the fifth finger flexor digitorum superficialis on grip strength. J Hand Ther 2003;16:245-8.

6. Kozin SH, Porter S, Clark P, Thoder JJ. The contribution of the intrinsic muscles to grip and pinch strength. J Hand Surg Am 1999;24:64-72.

7. Schrueders TAR, Stam HJ. Strength measurements of the lumbrical muscles. J Hand Ther 1996;9:303-5. 
8. Firrell JC, Crain GM. Which setting of the dynamometer provides maximal grip strength? J Hand Surg 1996;21A:397-401.

9. Fess EE. The effects of Jamar dynamometer handle position and test protocol on normal grip strength. J Hand Surg 1981;7:308-9.

10. Fess EE. Clinical Assessment Recommendations: 2. Chicago: American Society of Hand Therapists, 1992.

11. Li Z-M, Latash ML, Zatsiorsky VM. Force sharing among fingers as a model of the redundancy problem. Exp Brain Res 1998:119:276-86.
12. Kinoshita H, Murase T, Bandou T. Grip posture and forces during holding cylindrical objects with circular grips. Ergonomics 1996:39:1163-76.

13. Ohtsuki T. Inhibition of individual fingers during grip strength exertion. Ergonomics 1981:24:21-36.

14. LaStayo P, Hartzel J. Dynamic versus static grip strength: How grip strength changes when the wrist is moved, why dynamic grip strength may be a more functional measurement. J Hand Ther 1999;12:212-8. 